\title{
Measurements of the optical mirror coating properties.
}

\author{
V.B. Braginsky, A.A. Samoilenko \\ Dept. of Phys. Moscow State University \\ Moscow 119992, Russia.
}

October 22, 2018

\begin{abstract}
The results of measurement of optical mirror coating are presented. These results indicate that Standard Quantum Limit of sensitivity can be reached in the second stage of LIGO project if it is limited by thermoelastic noise in the coating only.
\end{abstract}

\section{Introduction.}

The sensitivity of terrestrial laser interferometer gravitational wave antennae [1] crucially depends on how the analysis of different kind of noises in parts of the set up, especially in the optical mirrors (test masses), is in-depth. Few years ago the analysis of the mirrors thermoelastic and thermorefractive noises "contribution" to the total noise budget was carried out [2, 3, 4]. The net result of this analysis is: these types of noises can make a significant "contribution" to the total noise if wrong material will be chosen for the mirrors. The analysis has also shown that fused silica has a serious advantage because of very small thermal expansion coefficient $\alpha_{\mathrm{Si}_{2}} \simeq 5 \times 10^{-7} \mathrm{~K}^{-1}$. At the same time the role of the mirrors coating has not been taken into account in this analysis. The coating of the mirror provides very important parameter of the mirror: the difference between the reflectivity $R$ and unity. The smaller is the value of $(1-R)$, the higher is the sensitivity of the antenna. It is worth noting that the smallness of $(1-R)$ also defines the sensitivity in many high resolution spectroscopical measurements.

The analysis of the role of thermoelastic noise produced due to the coating has been carried out recently by S.P. Vyatchanin and by one of this article authors and independently by M. Fejer and S. Rowan [5, 6]. The analytical formulas for the values of spectral densities of the noises in this publications are practically the same. The only difference is in dimensionless multiplier which depends on the ratio of the substrate (the mirror) and coating material constants. But substantial difference in [5] and [6] is in the numerical estimate of the spectral 
densities of the noise. This difference appears because the numerical value of the thermal expansion factor of tantalum pentoxide $\alpha_{\mathrm{Ta}_{2} \mathrm{O}_{5}}$ in [5] has been borrowed from the experiment by Inci [7]: $\alpha_{\mathrm{Ta}_{2} \mathrm{O}_{5}}=[-4.43 \pm 0.05] \times 10^{-5} \mathrm{~K}^{-1}$. This value is unexpectedly negative and large in contrast to $\left[\underline{6}\right.$, where the value of $\alpha_{\mathrm{Ta}_{2} \mathrm{O}_{5}}=+3.6 \times 10^{-6} \mathrm{~K}^{-1}$ has been borrowed from the measurement by Tien et al. [8] (positive value but without confidence limits). Due to this difference the predictions of the value of the noise produced by thermoelastic effect in [5] and [6] differs by one order. It is worth noting that this difference between these independent measurements may not be due to a mistake (or mistakes) of experiment but due to "pathological" properties of thin tantalum pentoxide layers. This harsh description of that solid belongs to S. Witcomb [9], who mentioned that the properties of $\mathrm{Ta}_{2} \mathrm{O}_{5}$ layer substantially depend on the chosen procedure of the layer deposition, and one may expect even mechanical and optical anisotropy of this type of layer.

These circumstances are the motivation for the experiments we have carried out and which are described below.

\section{The procedures and results of measurements.}

To measure the value of thermal expansion factor of tantalum pentoxide $\alpha_{\mathrm{Ta}_{2} \mathrm{O}_{5}}$ we used a well known method (see e.g. [8, 10]). This method is based on the measurement of bending of thin substrate plate (made of $\mathrm{Si}_{2}$ in our case) with coating, when plate is heated. The bending appears due to the difference $\alpha_{\mathrm{Ta}_{2} \mathrm{O}_{5}}-\alpha_{\mathrm{SiO}_{2}}$. If one end of the plate, which length is $l$, is rigidly attached to massive platform (which is not distorted by change of temperature) then one can observe the displacement $\Delta z$ of the plate free end due to the tension between the coating and the substrate that appears if the plate temperature changes by $\Delta T$. The value of $\alpha_{\mathrm{Ta}_{2} \mathrm{O}_{5}}$ may be calculated using the following formula which can be easily derived from formulas (30c) and (32b) of [10]:

$$
\alpha_{\mathrm{SiO}_{2}}-\alpha_{\mathrm{Ta}_{2} \mathrm{O}_{5}}=\frac{1}{3} \frac{E_{\mathrm{SiO}_{2}}\left(1-\nu_{\mathrm{Ta}_{2} \mathrm{O}_{5}}\right) d_{\mathrm{SiO}_{2}}^{2}}{E_{\mathrm{Ta}_{2} \mathrm{O}_{5}}\left(1-\nu_{\mathrm{SiO}_{2}}\right) d_{\mathrm{Ta}_{2} \mathrm{O}_{5}} l^{2}}\left[\frac{\Delta z}{\Delta T}\right]
$$

where $E_{\mathrm{SiO}_{2}}$ is the plate Young modulus, $E_{\mathrm{Ta}_{2} \mathrm{O}_{5}}$ is the $\mathrm{Ta}_{2} \mathrm{O}_{5}$ Young modulus, $d_{\mathrm{Ta}_{2} \mathrm{O}_{5}}$ is the total thickness of $\mathrm{Ta}_{2} \mathrm{O}_{5}$ coating, $d_{\mathrm{SiO}_{2}}$ is the thickness of the plate, $\nu_{\mathrm{Ta}_{2} \mathrm{O}_{5}}$ and $\nu_{\mathrm{SiO}_{2}}$ are the Poisson's ratios of $\mathrm{Ta}_{2} \mathrm{O}_{5}$ and plate solid respectively.

To reproduce the coating for a real mirror manufactured for the LIGO antenna in our tests we have prepared $100 \mu \mathrm{m}$ flat fused silica plates which were very well polished and have the shape of stripes $2 \mathrm{~cm}$ long and $1 \mathrm{~cm}$ wide. A set of such thin fused silica platesstripes was placed on a big relatively thick fused silica mini platform. The very ends of each stripe were rigidly attached to the platform. Due to the care of Dr. H. Armandula this

platform (with the plates-stripes on it) was placed into the same vacuum chamber together 
with big End Mirror for LIGO (10 cm thick, $25 \mathrm{~cm}$ in diameter), where the deposition of the multilayer coating was performed. Our fused silica plates-stripes "received" the same 19 pairs of quarter-wavelength $\mathrm{Ta}_{2} \mathrm{O}_{5}+\mathrm{Si} \mathrm{O}_{2}$ layers. Thus our plates-stripes were covered with the same coating (which has the thickness of $5.5 \mu \mathrm{m}$ and the total thickness of $\mathrm{Ta}_{2} \mathrm{O}_{5}$ $-2.2 \mu \mathrm{m})$ as the big mirror. This coating provides the value of $(1-R) \simeq 1 \times 10^{-5}$.

When the process of deposition was over and the plates-stripes were detached from the platform we have got the first (unexpected) experimental result: all plates-stripes were bent. The measured value of the curvature radius of these plates-stripes was $\simeq 11 \mathrm{~cm}$ (in the plane of long axis of the stripes). This bending remained unchanged many weeks after the detachment from the platform.

The main elements of the measuring set up are the two massive fused silica rectangular blocks which were rigidly attached to a heavy optical bench. A plate-stripe was rigidly clamped between two blocks. To evade damage due to the clamping a $15 \mu \mathrm{m}$ teflon film was inserted between plate-stripe and the surfaces of fused silica blocks. Power stabilized He-Ne laser, two lenses and photodiode were installed on the bench. This simple optical system permitted to measure $0.1 \mu \mathrm{m}$ displacement of the plate-stripe edge ("knife and slot" technique). Small box around a plate-stripe permitted to heat the plate-stripe by a slow stream of heated air.

Four sets of measurements with two different plates were carried out. The measurements with each plate were performed two times, second time' with the plate flipped up side down. The value of $[\Delta z / \Delta T]$ was obtained from the directly measured dependence of the edge displacement at the free end of plate-stripe on temperature. By using formula (11) with parameters $\alpha_{\mathrm{SiO}_{2}}=5.5 \times 10^{-7} \mathrm{~K}^{-1}, E_{\mathrm{SiO}_{2}}=7.2 \times 10^{11} \frac{\mathrm{erg}}{\mathrm{cm}^{3}}, \nu_{\mathrm{SiO}_{2}}=0.17, E_{\mathrm{Ta}_{2} \mathrm{O}_{5}}=$ $1.4 \times 10^{12} \frac{\mathrm{erg}}{\mathrm{cm}^{3}}, \nu_{\mathrm{Ta}_{2} \mathrm{O}_{5}}=0.23$ and measured $[\Delta z / \Delta T]$ the linear thermal expansion coefficient $\alpha_{\mathrm{Ta}_{2} \mathrm{O}_{5}}$ was estimated. Its value is equal to:

$$
\alpha_{\mathrm{Ta}_{2} \mathrm{O}_{5}}=[5 \pm 1] \times 10^{-6} \mathrm{~K}^{-1}
$$

\section{Conclusion.}

The obtained value of $\alpha_{\mathrm{Ta}_{2} \mathrm{O}_{5}}$ is evidently closer to the one obtained by Tien et al. [8] then to the one obtained by Inci [7. In the same time our value is almost two times larger then the smaller value [8]. In addition we have to note that our value of $\alpha_{\mathrm{Ta}_{2} \mathrm{O}_{5}}$ is based not only on the measured value of $\Delta z$ but also on assumption that the values of $E_{\mathrm{SiO}_{2}}$ and $E_{\mathrm{Ta}_{2} \mathrm{O}_{5}}$ (measured by other experimentalists) are correct. Taking into account that these values may be substantially different in thin layers and that the process of deposition may also cause changes of the Young moduli values (see e. g. [11]), we may conclude that the value of $\alpha_{\mathrm{Ta}_{2} \mathrm{O}_{5}}$ is between $3 \times 10^{-6} \mathrm{~K}^{-1}$ and $7 \times 10^{-6} \mathrm{~K}^{-1}$. The only "remedy" to 
solve this problem is the direct measurement of the mirror with coating surface fluctuations spectral density. At the same time the limits (from $3 \times 10^{-6} \mathrm{~K}^{-1}$ to $7 \times 10^{-6} \mathrm{~K}^{-1}$ ) permit to cancel the pessimistic prediction for the spectral density of the thermoelastic noise in the coating [5] which was based on Inci's value $\alpha_{\mathrm{Ta}_{2} \mathrm{O}_{5}}=[-4.43 \pm 0.05] \times 10^{-5} \mathrm{~K}^{-1}$. For example, the potentially achievable sensitivity for LIGO-II near the frequency of observation $f \simeq 10^{2} \mathrm{~Hz}$ may not be at the level of $S_{h} \simeq 1.5 \times 10^{-23} 1 / \sqrt{\mathrm{Hz}}$ (as in [5]) but between $0.6 \times 10^{-24} 1 / \sqrt{\mathrm{Hz}} \leqslant \mathrm{S}_{\mathrm{h}} \leqslant 1.4 \times 10^{-24} 1 / \sqrt{\mathrm{Hz}}$. It means that the projected sensitivity of LIGO-III (better than the Standard Quantum Limit) will be evidently not possible due to this noise.

To the above conclusive remarks we have to add that the "unpleasant discovery" i.e. the observation of the "frozen" bending of the plates-stripes with coating, which indicates the existence of mechanical stresses in the coating has to be regarded as strong argument in favor of direct measurement of the noises in coating, especially the measurement of $1 / f$ component.

The authors of this article are expressing their sincere gratitude to S.P. Vyatchanin and H. Armandula for their advice and direct help. This work was supported by NSF grant PHY-0098-715 and by Russian Ministry of Industry and Science.

\section{References}

[1] 1. Abramovici A.A. et al., LIGO: The laser interferometer gravitational-wave observatory. Science, v. 252, 1992, p. 325

[2] V.B. Braginsky, M.L. Gorodetsky, S.P. Vyatchanin, Phys. Lett. A 264, 1 (1999).

[3] Yu.T. Liu, K.S. Thorne, Phys. Rev. D62, 122002 (2002).

[4] V.B. Braginsky, M.L. Gorodetsky, S.P. Vyatchanin, Phys. Lett. A 271, 303-307 (2000).

[5] V.B. Braginsky, S.P. Vyatchanin, Thermodynamical fluctuation in optical mirror coatings. arXiv:cond-mat10302617, 3 Mar 2003.

[6] M.Fejer, S.Rowan - report at LSC meeting, Livingstone, March 18-21, 2003.

[7] Inci M.N. Simultaneous measurements of thermal optical and linear thermal expansion coefficients of $\mathrm{Ta}_{2} \mathrm{O}_{5}$ films, ICO 19, 25-30 August 2002, Firenze, Italy.

[8] Tien C.L. et al., Simultaneous determination of the thermal expansion coefficient and the elastic modulus of $\mathrm{Ta}_{2} \mathrm{O}_{5}$ thin film using phase shifting interferometry., J. of Mod. Opt., 2000, v. 47, no. 10, 1681-1691. 
[9] S.Whitcomb - private communication.

[10] P.H. Townsend et al., Elastic relationship in layered composite media with approximation for case of thin films on a thick substrate. J. Appl. Phys., v.62 (11), 1987, p.4438-4444.

[11] Martin P.J. et al., Mechanical and optical properties of thin films of tantalum oxide deposited by ion-assisted deposition, Mat. Res. Soc. Symp. Proc., v. 308, 1993, p.583588. 